International Journal of Pure and Applied Mathematics

Volume 86 No. 4 2013, 593-605

ISSN: $1311-8080$ (printed version); ISSN: 1314-3395 (on-line version)

url: http://www.ijpam.eu

doi: http://dx.doi.org/10.12732/ijpam.v86i4.1

\title{
A METHOD FOR INCORPORATING FRACTIONAL-ORDER DYNAMICS THROUGH PID CONTROL SYSTEM RETUNING
}

\author{
Emmanuel A. Gonzalez ${ }^{1,2,3}$ §, Concepción A. Monje ${ }^{4}$, \\ L’ubomír Dorčák ${ }^{5}$, Ján Terpák ${ }^{5}$, Ivo Petráš ${ }^{5}$ \\ ${ }^{1}$ Department of Computer Technology \\ College of Computer Studies \\ De La Salle University Manila 2401 Taft Ave. \\ Malate Manila 1004, PHILIPPINES \\ ${ }^{2}$ School of EECE \\ Mapua Institute of Technology \\ Muralla St., Intramuros Manila 1000, PHILIPPINES \\ ${ }^{3}$ Jardine Schindler Elevator Corporation \\ 8/F Pacific Star Bldg., Sen. Gil Puyat Ave. Cor. Makati Ave. \\ Makati City 1209, PHILIPPINES \\ ${ }^{4}$ Departamento de Ingeniería de Sistemas y Automática \\ Universidad Carlos III de Madrid \\ 28911, Legans Madrid, SPAIN \\ ${ }^{5}$ Institute of Control and Informatization of Production Processes \\ Faculty BERG \\ Technical University of Košice \\ B. Němcovej 3,042 00 Košice, SLOVAKIA
}

\begin{abstract}
Proportional-Integral-Derivative (PID) controllers have been the heart of control systems engineering practice for decades because of its simplicity and ability to satisfactory control different types of systems in different fields of science and engineering in general. It has receive widespread attention both in the academe and industry that made these controllers very mature and
\end{abstract}

Received: October 27, 2012

(c) 2013 Academic Publications, Ltd. url: www.acadpubl.eu

$\S$ Correspondence author 
applicable in many applications. Although PID controllers (or even its family counterparts such as proportional-integral $[\mathrm{PI}]$ and proportional-derivative [PD] controllers) are able to satisfy many engineering applications, there are still many challenges that face control engineers and academicians in the design of such controllers especially when guaranteeing control system robustness. In this paper, we present a method in improving a given PID control system focusing on system robustness by incorporating fractional-order dynamics through a returning heuristic. The method includes the use of the existing reference and output signals as well as the parameters of the original PID controller to come up with a new controller satisfying a given set of performance characteristics. New fractional-order controllers are obtained from this heuristic such as $\mathrm{PI}^{\lambda}$ and $\mathrm{PI}^{\lambda} \mathrm{D}^{\mu}$ controllers, where $\lambda, \mu \in(0,2)$ are the order of the integrator and differentiator, respectively.

AMS Subject Classification: 26A33, 37N35

Key Words: fractional-order systems, PID controllers, unity-feedback system

\section{Introduction and Problem Description}

We consider a problem of improving the robustness characteristics of a unityfeedback closed-loop system incorporating a stable plant $P(s)$ and a controller $C(s)$ of the PID family type, i.e. P, PI, PD, and PID, such as guaranteeing gain cross-over frequency and phase margin specifications, and robustness to system gain variations. Such integer-order PID family considered are also refered to as classical PID controllers in this paper. In [1], a practical approach was presented dealing with the closed-loop identification of a PID system through the identification of the parameters of a new controller $C_{R}(s)$, which are dependent on the reference input and output signals, and the original controller parameters. The method proposed in [1] has a distinguished feature of tuning a new controller that rely on the closed-loop model of the system and not of the process, which is also readily adaptable in the engineering context, i.e. no major modifications needed in the original closed-loop system. The idea is to determine the type of controller $C_{R}(s)$ best suited for its application and tuning of its parameters using any prefered means such as the well-known Ziegler-Nichols method [2]. Particularly in [1], the original controllers used on the synthesis method of $C_{R}(s)$ are based on the classical (integer-order) PI and classical PID approaches.

In this paper, we extend the work of [1] to incorporate fractional-order dynamics in the original closed-loop system using a retuning heuristic. Since it has 


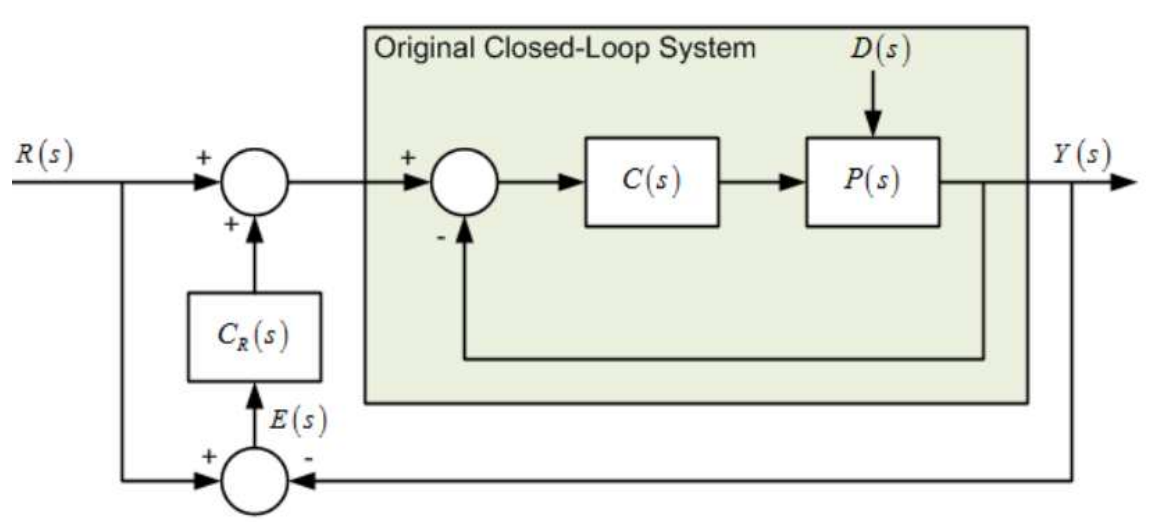

Figure 1: Original feedback control system with a PID family controller as $C(s)$

been proven in simulation and actual laboratory results that systems controlled by fractional-order controllers could yield outstanding results as compared to its integer-order counterparts. One example of such advantage is the ability to make the system robust having constant overshoots even if the gain is varied. Such advantage is achieved because of the ability of fractional-order systems to be set in such a way that its phase margin is invariant to gain changes. Furthermore, having such method could further enhance the properties of a closed-loop system [3]. Generally, the idea is to determine a new controller $C_{R}(s)$, in which the parameters are functions of the existing classical PID controller constants in $C(s)$ and to incorporate the new controller in the system without modifying the original system's internal architecture. Aside from this, the values of the new constants in $C_{R}(s)$ would also depend on the measured or calculated closedloop system model that will dictate the type of fractional-order controller to be used whether it is a fractional $\mathrm{PI}^{\lambda}$ or fractional $\mathrm{PI}^{\lambda} \mathrm{D}^{\mu}$ controller. Methods presented in $[4,5,6,7]$ can be used in such cases.

\section{Control System Retuning Architecture}

Figure 1 presents the original unity-feedback control system that is being considered in this paper with additional controller $C_{R}(s)$ appended resulting in a new system architecture. The plant $P(s)$ is assumed to be generally stable. A 


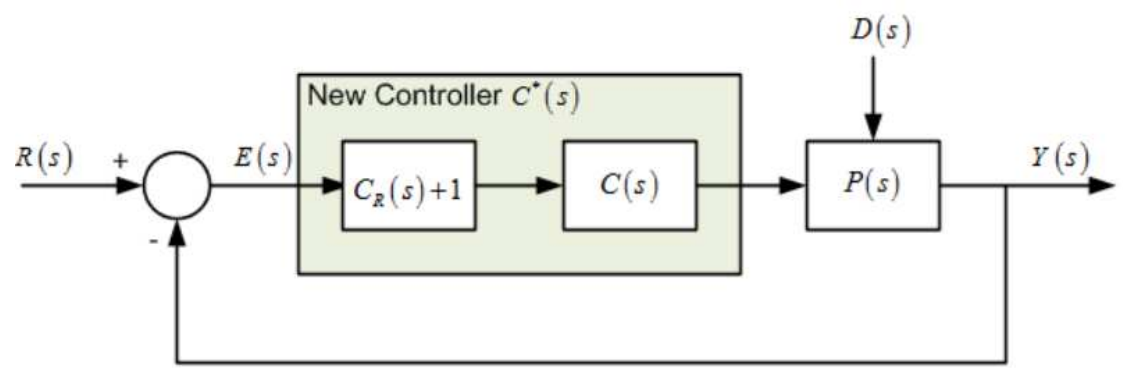

Figure 2: Equivalent feedback control system controller $C^{*}(s)$

simple time-delayed first-order system of the form

$$
P_{F O S T D}(s)=\frac{K}{T s+1} e^{-L s},
$$

or a generic time-delayed fractional-order system of the form

$$
P_{G F O S T D}(s)=\frac{K}{T s^{\delta}+1} e^{-L s},
$$

where $K, T, L, \delta>0$ without loss of generality can be assumed in this case. Furthermore, the controller in Figure 1 is considered to be of a classical PID family type, i.e. either a classical PI or classical PID controller having the forms

$$
C_{P I}(s)=K_{P}+\frac{K_{I}}{s},
$$

and

$$
C_{P I D}(s)=K_{P}+\frac{K_{I}}{s}+K_{D} s,
$$

respectively, where $K_{P}, K_{I}, K_{D}>0$.

In Figure $1, C_{R}(s)$ is a new controller that measures the input reference signal and the output signal in determining the error. This error is then processed by the new controller and is fed as part of the new reference signal to the original closed-loop system. The idea is to incorporate a new controller such that the internal architecture of the original feedback control system is not modified; hence, only the reference signal to the original closed-loop system is manipulated.

Using simple block diagram algebra, incorporating $C_{R}(s)$ into the system would result in an equivalent unity-feedback system depicted in Figure 2.

Given the original specifications of $C(s)$, the objective is to determine the appropriate parameters in $C_{R}(s)$ to be able to improve the robustness of the 
entire feedback control system. The new feedback control system controller $C_{P I, P I D}^{*}(s)=f\left(C_{P I, P I D}(s)\right)$ is seen to be also a function of the original classical PI and PID controller. Furthermore, the models of the new feedback control system controller is represented as

$$
C_{P I}^{*}(s)=K_{P}^{*}+\frac{K_{I}^{*}}{s^{\lambda}}
$$

and

$$
C_{P I D}^{*}(s)=K_{P}^{*}+\frac{K_{I}^{*}}{s^{\lambda}}+K_{D}^{*} s^{\mu},
$$

where $K_{P}^{*}, K_{I}^{*}, K_{D}^{*}>0$ and $0<\lambda, \mu<2$ are asssumed without loss of generality. Since the objective is to have a fractional-order PID controller, it is import that satisfy the conditions $0<\lambda, \mu<2$.

The determination of the parameters of the new controller

$$
C^{*}(s)=\left(C_{R}(s)+1\right) C(s)
$$

are presented in the next subsections.

\subsection{Classical PI to Fractional-Order $\mathrm{PI}^{\lambda}$ and $\mathrm{PI}^{\lambda} \mathrm{D}^{\mu}$ Controllers}

Lemma 1. Consider the controller of the form

$$
C_{R 1}(s)=\frac{K_{1} s^{\alpha}+\left(K_{0}-K_{P}\right) s-K_{I}}{K_{P} s+K_{I}},
$$

where it is assumed, without loss of generality that $K_{1}, K_{0}>0$ and $-1<\alpha<$ 1 , and the values of $K_{P}, K_{I}>0$ are obtained form the original classical PI controller in (2.3). The resulting fractional-order $P I^{\lambda}$ controller from a classical PI controller with parameters $K_{P}$ and $K_{I}$ will have the following coefficients for $0<\lambda<2$ :

$$
K_{P}^{*}=K_{0}
$$

and

$$
K_{I}^{*}=K_{1}
$$

The order of integration is

$$
\lambda=1-\alpha .
$$

Proof. Equation (2.7) directly results in

$$
C^{*}(s)=\left(C_{R 1}(s)+1\right) C(s)
$$




$$
\begin{aligned}
= & \frac{1}{K_{P} s+K_{I}} \\
& \times\left(K_{1} s^{\alpha}+\left(K_{0}-K_{P}\right) s-K_{I}\right. \\
& \left.\quad+K_{P} s+K_{I}\right) \\
& \times \frac{K_{P} s+K_{I}}{s} \\
= & K_{0}+\frac{K_{1}}{s^{1-\alpha}}=K_{P}^{*}+\frac{K_{I}^{*}}{s^{\lambda}} \cdot \square
\end{aligned}
$$

Lemma 1 shows that the new parameters of $C^{*}(s)$ can explicitly be determined as long as the values $K_{P}, K_{I}$, and $\alpha$ are given. It is also important to note that the controller $C_{R 1}(s)$ cancels out the effects of $K_{I}$ and $K_{P}$ in the original feedback control system and introduces the fractional dynamics of $K_{I}^{*} s^{-\lambda}$. The new proportional constant $K_{P}^{*}$ is directly dictated by $K_{0}$. "Full control" is achieved because the dynamics controller is solely dictated by $K_{1}$ and $K_{0}$. The resulting controller has three degrees of freedom (3-DOF) which will now make it possible to satisfy three robustness criteria, which cannot be done by a classical PI controller having only two degrees of freedom, i.e. $K_{P}$ and $K_{I}$ only.

Lemma 2. Consider the controller of the form

$$
C_{R 2}(s)=\frac{K_{2} s^{\beta}+K_{1} s^{\alpha}+\left(K_{0}-K_{P}\right) s-K_{I}}{K_{P} s+K_{I}},
$$

where it is assumed, without loss of generality that $K_{0}, K_{1}, K_{2}>0$ with $-1<$ $\alpha<1$ and $1<\beta<2$ (higher-order). The resulting fractional-order $P I^{\lambda} D^{\mu}$ controller from a classical PI controller with parameters $K_{P}$ and $K_{I}$ will have the following coefficients for $0<\lambda<2$ :

$$
\begin{aligned}
& K_{P}^{*}=K_{0}, \\
& K_{I}^{*}=K_{1},
\end{aligned}
$$

and

$$
K_{D}^{*}=K_{2}
$$

The orders of integration and differentiation are

$$
\lambda=1-\alpha
$$

and

$$
\mu=\beta-1
$$

respectively. 
Proof. Equation (2.7) directly results in

$$
\begin{aligned}
C^{*}(s)= & \left(C_{R 2}(s)+1\right) C(s) \\
= & \frac{1}{K_{P} s+K_{I}} \\
& \times\left(K_{2} s^{\beta}+K_{1} s^{\alpha}+\left(K_{0}-K_{P}\right) s-K_{I}\right. \\
& \left.\quad+K_{P} s+K_{I}\right)\left(\frac{K_{P} s+K_{I}}{s}\right) \\
= & K_{0}+\frac{K_{1}}{s^{1-\alpha}}+K_{2} s^{\beta-1}=K_{P}^{*}+\frac{K_{I}^{*}}{s^{\lambda}}+K_{D}^{*} s^{\mu} .
\end{aligned}
$$

The controller $C_{R 2}(s)$ in (2.12) enables the designer to have full control of the feedback control system be totally eliminating the effects of the original classical PI controller and introducing the fractional dynamics of the resulting fractional-order $\mathrm{PI}^{\lambda} \mathrm{D}^{\mu}$ controller. The resulting fractional-order $\mathrm{PI}^{\lambda} \mathrm{D}^{\mu}$ has five degrees of freedom which means that five robustness criteria can be used.

\subsection{Classical PID to Fractional-Order $\mathrm{PI}^{\lambda} \mathrm{D}^{\mu}$ Controllers}

Lemma 3. Consider the controller of the form

$$
C_{R 3}(s)=\frac{K_{2} s^{\beta}+K_{1} s^{\alpha}-K_{D} s^{2}+\left(K_{0}-K_{P}\right) s-K_{I}}{K_{D} s^{2}+K_{P} s+K_{I}},
$$

where it is assumed, without loss of generality that $K_{1}, K_{2}>0$ with $-1<\alpha<1$ and $1<\beta<2$ (higher-order). The resulting fractional-order $P I^{\lambda} D^{\mu}$ controller from a classical PID controller with parameters $K_{P}, K_{I}$, and $K_{D}$ will have the following coefficients for $0<\lambda<2$ :

$$
\begin{aligned}
K_{P}^{*} & =K_{0}, \\
K_{I}^{*} & =K_{1},
\end{aligned}
$$

and

$$
K_{D}^{*}=K_{2} .
$$

The orders of integration and differentiation are

$$
\lambda=1-\alpha,
$$

and

$$
\mu=\beta-1,
$$

respectively. 
Proof. Equation (2.7) directly results in

$$
\begin{aligned}
C^{*}(s)= & \left(C_{R 3}(s)+1\right) C(s) \\
= & \frac{1}{K_{D} s^{2}+K_{P} s+K_{I}} \\
& \times\left(K_{2} s^{\beta}+K_{1} s^{\alpha}-K_{D} s^{2}+\left(K_{0}-K_{P}\right) s-K_{I}\right. \\
& \left.\quad+K_{P} s+K_{I}\right)\left(\frac{K_{D} s^{2}+K_{P} s+K_{I}}{s}\right) \\
& \quad K_{0}+\frac{K_{1}}{s^{1-\alpha}}+K_{2} s^{\beta-1}=K_{P}^{*}+\frac{K_{I}^{*}}{s^{\lambda}}+K_{D}^{*} s^{\mu} .
\end{aligned}
$$

The controller $C_{R 3}(s)$ in (2.18) also enables the designer to have full-control of the feedback control system be totally eliminating the effects of the original classical PID controller and introducing the fractional dynamics of the resulting fractional-order $\mathrm{PI}^{\lambda} \mathrm{D}^{\mu}$ controller.

Corollary 4. If $1<\lambda<2$, then the term $K_{1} s^{\alpha}$ becomes an integral term with an order of $|\alpha|$.

Proof. It can easily be seen from the previous lemmata that $\alpha=1-\lambda$ will result to a negative value.

In the case where $1<\lambda<2$, the fractional-order integral part of the controller is considered to be of "higher-order."

\section{Retuning Heuristic}

The retuning process basically ends up with the identificaiton of the parameters of the new controller $C_{R}(s)$ given the parameters of the original controller $C(s)$ and the desired new PID controller constants $C^{*}(s)$. For a well-determined original closed-loop system, the heuristic is presented as follows:

1. Determine how many robustness criteria are to be satisfied. If two or three criteria are to be satisfied, then a new fractional-order $\mathrm{PI}^{\lambda}$ controller will suffice which can be used regardless if the original controller is classical PI or classical PID. If four or five criteria are to be satisfied, then a new fractional-order $\mathrm{PI}^{\lambda} \mathrm{D}^{\mu}$ controller can be used. Examples of robustness criteria are : a) phase margin specification

$$
\arg \left(C^{*}\left(j \omega_{c g}\right) P\left(j \omega_{c g}\right)\right)=-\pi+\varphi_{m},
$$


where $\omega_{c g}$ is the gain cross-over frequency; b) gain cross-over frequency specification $\left|C^{*}\left(j \omega_{c g}\right) P\left(j \omega_{c g}\right)\right|=1$; c) robustness to variations in the gain of the plant $\left.\left(d / d \omega_{c g}\right) C^{*}\left(j \omega_{c g}\right) P\left(j \omega_{c g}\right)=0 ; \mathrm{d}\right)$ high-frequency noise rejection where the complementary sensitivity function is constratined at $|T(j \omega)| \leq A$ for a certain range of frequencies $\omega \geq \omega_{t} \mathrm{rad} / \mathrm{sec}$; e) ensuring output disturbance rejection where the sensitivity function is constratined at $|S(j \omega)| \leq B$ for a certain range of frequencies $\omega \leq \omega_{s} \mathrm{rad} / \mathrm{sec}$; and $\mathrm{f}$ ) steady-state error cancelation $\lim _{t \rightarrow \infty} y(t)=0$.

2. Determine the parameters of $C^{*}(s)$ based on the plant's parameters through the use of any existing tuning or optimization method. Tuning methods for fractional-order $\mathrm{PI}^{\lambda}$ can be used such as $[5,6]$, while $[4,7]$ can be used for the tuning of fractional-order $\mathrm{PI}^{\lambda} \mathrm{D}^{\mu}$ controllers.

3. Calculate the coefficients of $C_{R}(s)$ through the lemmata presented in the previous section.

\section{Numerical Examples}

\subsection{Example 1: Unmanned Aerial Vehicle (UAV) [5]}

Consider an unmanned aerial vehicle (UAV) which is represented by a timedelayed first-order system (2.1) having the transfer function

$$
P(s)=\frac{0.9912}{0.3414 s+1} e^{-0.2793 s}
$$

with $K=0.9912, T=0.3414$, and $L=0.2793$. The original PI controller in the closed-loop system has the transfer function

$$
C(s)=0.37+\frac{1.3542}{s}
$$

where $K_{P}=0.37$ and $K_{I}=1.3542$. The objective is to satisfy three robustness criteria: a) phase margin set at $\varphi_{m}=65$ degrees at the gain cross-over frequency; b) gain cross-over frequency set at $\omega_{c g}=1.3 \mathrm{rad} / \mathrm{s}$; and c) robustness to gain variations.

1. Since only three robustness criteria are chosen, using (2.8) in Lemma 1 will already suffice. 
2. Using [5], the obtained parameters of the new fractional-order $\mathrm{PI}^{\lambda}$ controller are $K_{P}^{*}=0.7092, K_{I}^{*}=1.4868$, and $\lambda=1.2029$.

3. The coefficients of (2.8) can then be calculated through (2.9)-(2.11): $K_{0}=$ $K_{P}^{*}=0.7092, K_{1}=K_{I}^{*}=1.4868$, and $\alpha=1-\lambda=-0.2029$. Since $1<\lambda<2$, Corollary 4 will apply. This will then result in the controller

$$
\begin{aligned}
C_{R}(s)= & \frac{K_{1} s^{\alpha}+\left(K_{0}-K_{P}\right) s-K_{I}}{K_{P} s+K_{I}} \\
= & \frac{1}{0.37 s+1.3542} \\
& \times\left(1.4868 s^{-0.2029}\right. \\
& \quad+(0.7092-0.37) s \\
= & \frac{-1.3542)}{0.3392 s-1.3542+1.4868 s^{-0.2029}} \\
= & \frac{N(s)}{s^{0.2029}(0.37 s+1.3542)},
\end{aligned}
$$

where $N(s)=0.3392 s^{1.2029}-1.3542 s^{0.2029}+1.4868$.

\subsection{Example 2: Pressurized Heavy Water Reactor (PHWR) [4]}

Consider the control of a PHWR represented by a generic time-delayed fractionalorder system (2.2) with a plant transfer function of

$$
P(s)=\frac{195.0736}{1.0006 s^{1.057}+1} e^{-0.0934 s}
$$

having the values $K=195.0736, T=1.0006, L=0.0934$, and $\delta=1.057$. The original classical PID controller is defined by

$$
C(s)=0.0052+\frac{0.0051}{s}+0.00007 s
$$

where the following values are obtained: $K_{P}=0.0052, K_{I}=0.0051$, and $K_{D}=$ 0.00007. The objective is to satisfy the following robustness criteria: a) a certain phase margin set in [4]; b) gain cross-over frequency set at $\omega_{c g}=1.0 \mathrm{rad} / \mathrm{s}$; c) robustness to gain variations; d) a certain high-frequency noise rejection value set in [4] with cut-off at $\omega_{t}=100 \mathrm{rad} / \mathrm{s}$; and e) ensuring output disturbance rejection with a certain value in [4] having a cut-off at $\omega_{s}=0.01 \mathrm{rad} / \mathrm{s}$. 
1. Since five robustness criteria are chosen, using (2.18) in Lemma 3 to assume full control will suffice.

2. Using [4], the obtained parameters of the new fractional-order $\mathrm{PI}^{\lambda} \mathrm{D}^{\mu}$ controller are $K_{P}^{*}=0.0006, K_{I}^{*}=0.0052, K_{D}^{*}=0.0049, \lambda=1.0137$, and $\mu=0.1067$.

3. The coefficients of (2.18) can then be calculated through (2.19)-(2.23): $K_{0}=K_{P}^{*}=0.0006, K_{1}=K_{I}^{*}=0.0052, K_{2}=K_{D}^{*}=0.0049, \alpha=1-\lambda=$ -0.0137 , and $\beta=\mu+1=1.1067$. Since $1<\lambda<2$, Corollary 4 will apply. This will then result in the controller

$$
\begin{aligned}
C_{R}(s)= & \frac{1}{K_{D} s^{2}+K_{P} s+K_{I}} \\
& \times\left(K_{2} s^{\beta}+K_{1} s^{\alpha}-K_{D} s^{2}\right. \\
& \left.\quad+\left(K_{0}-K_{P}\right) s-K_{I}\right)
\end{aligned}
$$

where

$$
\begin{aligned}
N(s)= & -s^{2.0137}+70.0 s^{1.1204}-65.71 s^{1.0137} \\
& -72.86 s^{0.0137}+74.29
\end{aligned}
$$

and

$$
\begin{aligned}
D(s)= & s^{2.0137}+74.29 s^{1.0137} \\
& +72.86 s^{0.0137}
\end{aligned}
$$

\section{Conclusion}

We have presented in this paper a method in improving a given classical PID family control system through a retuning heuristic focusing on the achieving 
various robustness criteria. The choice of controller would highly depend on the number of robustness criteria to satisfy. The heuristic is a three-step process that would require: 1) the identification or selection of robustness criteria to satisfy a certain set of specifications; 2) obtaining the parameters of the fractional-order $\mathrm{PI}^{\lambda}$ or fractional-order $\mathrm{PI}^{\lambda} \mathrm{D}^{\mu}$ controller based on any existing tuning method; and 3) the calculation of interal parameters of the controller $C_{R}(s)$. The heuristic is tested on a time-delayed first-order system (2.1) and on a generic fractional-order system with time delay (2.2).

The configuration in Figure 1 is somewhat straightforward as it does not need for a manipulation in the original closed-loop systems architecture. Such configuration is of advantage to the control engineer in the assumption that the reference input signal, actual output signal, and control signal to the original closed-loop system are easily accessible and controllable.

Finally, it is observerd that the resulting controllers in the previous lemmata have multiple terms, some of which having fractional-degrees. One approach in the implementaiton of such controllers is to convert these controllers into fraction expansions. For example, the controller (4.1) in Example 1 can be expanded as

$$
\begin{aligned}
C_{R}(s)= & \frac{0.3392 s^{1.2029}}{0.37 s^{1.2029}+1.3542^{0.2029}} \\
& -\frac{1.3452 s^{0.2029}}{0.37 s^{1.2029}+1.3542^{0.2029}} \\
& +\frac{1.4868}{0.37 s^{1.2029}+1.3542^{0.2029}}
\end{aligned}
$$

which is in the form of parallel systems. Such approach can be used especially if the implementation is done through analog circuitry. Infinite Impulse Response (IIR) architecture can alternately by used for digital control applications using various discretization methods available in literature such as bilinear transformation.

\section{Acknowledgments}

This work was partially supported by grant VEGA 1/0729/12 from the Slovak Grant Agency for Science in Slovakia. 


\section{References}

[1] H. M. Son, Retuning of PI/PID controllers based on closed-loop model, The AUN/SEED-Net Fieldwise Seminar on Control Engineering, Montien Hotel, Bangkok, Thailand, Mar. 16-17 (2006), Session 1-4.

[2] A. O'Dwyer, Handbook of PI and PID Controller Tuning Rules, 3rd. ed., Imperial College Press (2008).

[3] C. A. Monje, YQ. Chen, B. M. Vinagre, D. Xue, V. Feliu, Fractional-Order Systems and Controls: Fundamentals and Applications, London: SpringerVerlag (2010)

[4] Sa. Das, Sh. Das, A. Gupta, Fractional order modeling of a PHWR under step-back condition and control of its global power with a roust PID controller, IEEE Transactions on Nuclear Science, 58, No. 5, October (2011), 2431-2441.

[5] Y. Luo, H. Chao, L. Di, Y.Q. Chen, Lateral directional fractional order $(\mathrm{PI})^{\alpha}$ control of a small fixed-wing unmanned aerial vehicles: controller designs and flight tests, IET Control Theory and Applications, 5, Iss. 18 (2011), 2156-2167.

[6] Y. Luo YQ. Chen, Stabilizing and robust FOPI controller synthesis for first order plus time delay system, 50th IEEE Conference on Decision and Control and European Control Conference (CDC-ECC), Orlando, FL, USA, December 12-15 (2011), pp. 2040-2045.

[7] D. Xue, C. Zhao, YQ. Chen, Fractional order PID control of a DC-motor with elastic shaft: a case study, Proceedings of the 2006 American Control Conference, Minneapolis, Minnesota, USA, June 14-16 (2006) ThB11.5, 3182-3187. 
